\title{
RENDIMIENTO HISTÓRICO DE LAS ASIGNATURAS EL SER HUMANO EN SU ENTORNO Y GLOBALIZACIÓN Y AMBIENTE EN EL PERIODO 2007-2015
}

HISTORICAL PERFORMANCE OF THE SUBJECTS THE HUMAN BEING IN ITS ENVIRONMENT AND GLOBALIZATION AND ENVIRONMENT OF THE PERIOD 2007-2015

\section{Verónica Bonilla-Villalobos ${ }^{1}$ vbonilla@uned.ac.cr Universidad Estatal a Distancia, Costa Rica}

Volumen 8, Número 2

30 de noviembre del 2017

pp. $158-177$

Recibido: 4 de abril del 2017

Aprobado: 4 de agosto del 2017

\footnotetext{
1 Verónica Bonilla Villalobos, Encargada de Cátedra de Producción Sustentable, Escuela de Ciencias Exactas y Naturales, Universidad Estatal a Distancia, Costa Rica. Correo electrónico: vbonilla@uned.ac.cr.
}

Rendimiento histórico de las asignaturas Globalización y Ambiente y del El Ser Humano en su

Entorno de los años 2007-2015.

Verónica Bonilla-Villalobos

DOI: http://dx.doi.org/10.22458/caes.v8i2.1923

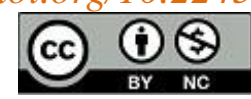

Artículo protegido por licencia Creative Commons 


\section{Resumen}

El artículo se enfoca en sistematizar los listados históricos de matrícula de los estudiantes de las asignaturas El Ser Humano en su Entorno y Globalización y Ambiente en el periodo 2007 y 2012, respectivamente. El objetivo de la investigación es conocer el rendimiento estudiantil y el porcentaje de aprobados y reprobados por año, identificando así el comportamiento de estas dos materias pertenecientes al Plan de Estudios Generales. Se realiza una revisión de los atestados correspondiente a la matrícula, para evidenciar los estudiantes aprobados, reprobados y otros rubros donde se contabiliza retiros justificados, injustificados (nsp), entre otros, paralelamente se hace una revisión de las orientaciones académicas de todos los periodos para contabilizar la cantidad de instrumentos de evaluación de los aprendizajes que se utilizan en cada cuatrimestre. Como resultado general se identificó que el promedio de aprobación oscila entre $68 \%$ y $70 \%$; en cuanto al promedio de estudiantes reprobados, van entre $23 \%$ y $20 \%$; los datos se relacionan con la cantidad de actividades sumativas utilizadas por cuatrimestre. Se concluye que no hay una relación directa con la cantidad de instrumentos y el porcentaje de aprobados y reprobados; además, el porcentaje de reprobados es aceptable debido a que en su mayoría los estudiantes son de primer ingreso, lo que significa el primero contacto con un sistema educativo a distancia. Con el fin de mejorar el rendimiento académico se recomienda aplicar herramientas para conocer la parte social del estudiantado; además, se sugiere utilizar la experiencia universitaria con el uso de las TIC como proyecto piloto en algunos centros universitarios.

Palabras clave: Rendimiento académico, pedagogía, evaluación pedagógica, estudios humanistas

\section{Abstract}

This article focuses on systematizing the historical enrollment lists of the students of the subjects Human Being in its Environment and Globalization and Environment between 2007 and 2015. The objective of the research is to know about student performance and the percentage of approval and failing per year, in these two subjects pertaining to the General Study Plan. It was made a review of the grades, justified and unjustified withdrawals and no shows, among others, in order to show the students' academic success; at the same time it was made a review of the academic orientations of all periods to account the number of evaluation instruments that are used in each quarter. As a general result it was identified that the average approval ranges from $68 \%$ to $70 \%$ and the average number of students that fail ranges between $23 \%$ and $20 \%$; these data are related to the sum of summation activities used per term. As a conclusion, there is no direct relation with the number of instruments and the

Rendimiento histórico de las asignaturas Globalización y Ambiente y del El Ser Humano en su

Entorno de los años 2007-2015.

Verónica Bonilla-Villalobos

DOI: http://dx.doi.org/10.22458/caes.v8i2.1923

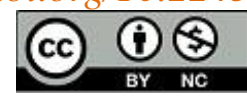

Artículo protegido por licencia Creative Commons 
percentage of approved and failed students; In addition, the percentage of failures is considered acceptable because the majority of students were freshmen, which means these subjects were the first contact with a distance education system, different from the classroom system. It is recommended to apply tools to know the social situation of the students in order to improve academic performance; in addition, it is suggested to use the university experience with the use of digital tools by implementing a pilot project in some university centers.

Keywords: Academic achievement, pedagogy, educational assessment, humanities education

\section{Introducción}

El programa de Estudios Generales, código 00, constituyen un programa académico que brinda un conjunto de asignaturas de carácter humanístico, enmarcado dentro de la oferta regular que tiene la Universidad para sus estudiante, programa adscrito a la Escuela de Ciencias Sociales y Humanidades (ECSH); las asignaturas en las que nos vamos a enfocar en este artículo son Globalización y Ambiente, código 076 y El Ser Humano en su Entorno código 073 (UNED, 2014).

Ambas materias se encuentran adscritas a la Escuela de Ciencias Exactas y Naturales, a partir del II cuatrimestre de 2014 pasan a formar parte de la Cátedra Sociedad y Ambiente, de la carrera de Manejo de Recursos Naturales; asimismo, actualmente se ofertan en la modalidad tradicional (es decir, no tiene entorno virtual); con una matrícula cuatrimestral de aproximadamente 1100 estudiantes por asignatura.

\section{Rendimiento histórico de las asignaturas Globalización y Ambiente y del EI Ser Humano en su \\ Entorno de los años 2007-2015. \\ Verónica Bonilla-Villalobos \\ DOI: http://dx.doi.org/10.22458/caes.v8i2.1923}

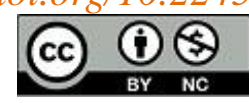


La Cátedra da inicio en el segundo cuatrimestre de 2014 (creación de la cátedra), los cursos están completamente listos para ser impartidos; bajo esta premisa, se imparte el curso bajo la modalidad tradicional con una evaluación sumativa de dos pruebas escritas ordinarias y un proyecto de investigación para un $100 \%$ y con un total de 31 profesores tutores distribuidos en todo el país.

Se identifica por medio de las reuniones de cátedra la necesidad de mejorar la calidad y eficacia de los cursos, una de las preocupaciones mayores por parte de los tutores y de la cátedra es la gran cantidad de estudiante que reprueba el curso, las bajas calificaciones especialmente en el trabajo de investigación, bajo el enfoque de Vygostsky, según Fallas y Trejos (2013), sobre construcción colectiva y los procesos de apropiación de los saberes y de los instrumentos de mediación, se estimula la participación del cuerpo docente de la cátedra para generar estrategias de mejora.

Aunque se trabaja ampliamente en el proceso formativo y en las actividades evaluativas de los aprendizajes, no se puede corroborar el nivel de aprendizaje del estudiante, pues no existe una retroalimentación eficiente debido a que no hay una interacción directa con los estudiantes.

A raíz de esta preocupación, para el III cuatrimestre de 2014, se modifica el modelo de evaluación: dos pruebas escritas ordinarias; un anteproyecto y un proyecto de investigación para un total de $100 \%$. Según Driscoll (2005), las estrategias asociadas al paradigma construccionista consideran que los conocimientos son construidos por el aprendiz, conforme éste trata de dar sentido a los procesos; a partir de esta premisa que toma la decisión de modificar el modelo de evaluación.

Rendimiento histórico de las asignaturas Globalización y Ambiente y del El Ser Humano en su

Entorno de los años 2007-2015.

Verónica Bonilla-Villalobos

DOI: http://dx.doi.org/10.22458/caes.v8i2.1923

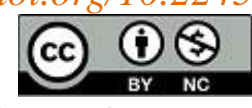

Artículo protegido por licencia Creative Commons 
El anteproyecto viene a jugar un papel formativo y de retroalimentación para que el estudiante pueda optar por corregir y mejorar su nota y presentar el proyecto final con menos errores, sin embargo, se presentan varios problemas:

1. Se identifica que el estudiante no lee las instrucciones dadas en la orientación académica en general.

2. El estudiante tiene dificultades a la hora de definir de que es un anteproyecto, (aunque en la orientación se explique).

3. Dificultad en seguir instrucciones dadas en la orientación académica.

4. Se identifica que algunos estudiantes no presentan el anteproyecto y no se está cumpliendo el objetivo de retroalimentación y aprendizaje.

5. En casi todos los casos no se realizan los cambios y las observaciones dadas por el profesor-tutor en el anteproyecto, para que su proyecto final mejore.

Debido a que se identificó que el cambio de los instrumentos no fue tan significativo y más bien pudo provocar bajas calificaciones, dentro de las conclusiones que llevamos a cabo se señala que posiblemente se da por el nivel de aprendizaje del estudiante, por ser de primer ingreso y la mayoría recién termina la secundaria; por tanto, Fallas y Trejos (2003) dicen que, según la teoría de Bruner, se requiere utilizar estrategias motivacionales como un factor en el aprendizaje para que el estudiante se apropie de su saber.

Según la teoría de Jean Piaget, la adquisición del conocimiento es un proceso continuo de autoconstrucción y que el estudiante integra la nueva información a los esquemas previos construidos; por lo tanto, para el año 2015 se plantea nuevamente un cambio en las actividades evaluativas del estudiante; con el fin de evaluar; el proceso de construcción del aprendizaje.

\section{Rendimiento histórico de las asignaturas Globalización y Ambiente y del El Ser Humano en su \\ Entorno de los años 2007-2015. \\ Verónica Bonilla-Villalobos \\ DOI: http://dx.doi.org/10.22458/caes.v8i2.1923}

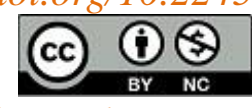

Artículo protegido por licencia Creative Commons 
Nolla (1998) menciona que la calidad de una asignatura depende de la selección de los elementos y de la valoración del carácter de estos, lo cual requiere no sólo medir los resultados obtenidos, sino que contempla la recopilación de información sobre los recursos y procesos que contribuyen a modificar los resultados, por ende la importancia de conocer la trayectoria histórica de estas asignaturas del 073 y 076 para visualizar de forma cualitativa y cuantitativa en la tasa de rendimiento y la tasa de éxito.

Colmenares y Delgado (2008) hacen referencia a que se presenta una influencia en el porcentaje de estudiantes reprobados, según su estado motivacional ya que afecta la vida de las personas y en particular su desempeño y prosecución en el plano escolar, por tanto, podría ser una de la consecuencias de la pérdidas de los asignaturas, para lo cual se deberán de realizar otros estudios para identificar el supuesto.

Rodríguez y Vindas (2005). hacen referencia a que la deserción estudiantil es uno de los aspectos que se manifiesta en el sistema educativo a distancia con un costo social elevado, es un fenómeno de carácter colectivo, en el cual los individuos, una vez que logran insertarse en el sistema de educación, abandonan el proceso formal sin completar el ciclo respectivo, debido a causas endógenas y exógenas al mismo sistema; con esta investigación se indaga el porcentaje de deserción en las asignaturas 073 y 076 pertenecientes a la Escuela de Estudios Generales.

Castaño, Gallón, Gómez, Vásquez (2008) señalan la importancia de que las instituciones de educación superior, en particular las públicas, emprendan campañas pedagógicas que recalquen la importancia de terminar los estudios en el tiempo teóricamente establecido para

Rendimiento histórico de las asignaturas Globalización y Ambiente y del El Ser Humano en su

Entorno de los años 2007-2015.

Verónica Bonilla-Villalobos

DOI: http://dx.doi.org/10.22458/caes.v8i2.1923

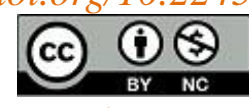

Artículo protegido por licencia Creative Commons 
completar los programas académicos, enfatizando en los costos implicados, tanto para los estudiantes y sus familias como para las instituciones y la sociedad en general.

\section{Metodología}

Trayectoria histórica de la asignatura: Se analizó información correspondiente a matrícula y oferta de las asignaturas EI Ser Humano y su Entorno y Globalización y Ambiente, desde el 2007 y hasta el 2015, con el fin de determinar la cantidad de estudiantes matriculados y la periodicidad con la que oferta al año.

Seguidamente, se observó la cantidad de actividades evaluativas utilizadas en cada asignatura (2007-2015), relacionando la cantidad de actividades y el rendimiento académico; esto se llevó a cabo mediante la revisión de la información contenida en las orientaciones académicas y la comparación con los rendimientos obtenidos en cada cuatrimestre que se ofertó.

Análisis del rendimiento de los estudiantes: Mediante la oficina de Registro de la Universidad, se obtienen los listados de matrícula con los históricos de las asignaturas 076 y 073 con el fin de identificar el rendimiento de los estudiantes; se solicita el siguiente detalle: total de estudiantes matriculados, aprobados, reprobados, reconocidas, retiro autorizado, retiro injustificado (nsp) y en curso.

Se analizan los datos tomando como variables independientes el año, el centro universitario y los cuatrimestres donde se ofertan las asignaturas; como variables dependientes, las condiciones de los estudiantes con el objetivo de identificar el rendimiento en ambos cursos.Resultados

\section{Rendimiento histórico de las asignaturas Globalización y Ambiente y del EI Ser Humano en su \\ Entorno de los años 2007-2015. \\ Verónica Bonilla-Villalobos \\ DOI: http://dx.doi.org/10.22458/caes.v8i2.1923}

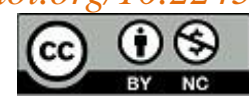


1. Trayectoria histórica de la asignatura

a. Oferta de las asignaturas por cuatrimestre 2007-2015

La asignatura El Ser Humano en su Entorno se oferta por primera vez en el año 2007, se evidencian variaciones en cuanto a su oferta académica cuatrimestral; además, se observa el aumento significativo de la matrícula por año (cuadro 1).

Cuadro 1. Oferta de la asignatura El Ser Humano en su Entorno 2007 - 2015

\begin{tabular}{c|c|c|c|c}
\hline $\begin{array}{c}\text { EI Ser Humano en su } \\
\text { Entorno }\end{array}$ & \multicolumn{4}{c}{ Cuatrimestre que se imparte } \\
\hline Año & Cant. Est. & I & II & III \\
\hline 2007 & 32 & - & - & X \\
2008 & 114 & X & - & X \\
2009 & 121 & X & - & X \\
2010 & 326 & - & X & - \\
2011 & 1980 & - & X & X \\
2012 & 2200 & - & X & X \\
2013 & 3090 & $X$ & $X$ & $X$ \\
2014 & 3011 & $X$ & $X$ & X \\
2015 & 3040 & $X$ & $X$ & X \\
\hline
\end{tabular}

Fuente: elaboración propia, datos facilitados por CIEI, UNED, 2015.

En cuanto a la asignatura Globalización y Ambiente, se ofrece por primera vez a los estudiantes en el año 2012 en el III cuatrimestre; durante el año 2013 se imparte el II y III

\section{Rendimiento histórico de las asignaturas Globalización y Ambiente y del El Ser Humano en su \\ Entorno de los años 2007-2015. \\ Verónica Bonilla-Villalobos \\ DOI: http://dx.doi.org/10.22458/caes.v8i2.1923}

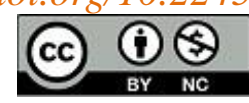


cuatrimestre y mientras en el 2015 los tres cuatrimestres, al igual que la asignatura anterior se observa un aumento en la matrícula cada año de ofertada (cuadro 2).

Cuadro 2. Oferta de la asignatura Globalización y Ambiente 2012-2015

\begin{tabular}{c|c|c|c|c}
\hline \multicolumn{2}{c}{$\begin{array}{c}\text { Globalización y } \\
\text { Ambiente }\end{array}$} & \multicolumn{3}{c}{$\begin{array}{c}\text { Cuatrimestre que se } \\
\text { imparte }\end{array}$} \\
\hline Año & Cant. Est. & I & II & III \\
\hline 2012 & 407 & - & - & X \\
2013 & 1586 & X & X & - \\
2014 & 2510 & X & X & X \\
2015 & 2544 & X & X & X \\
\hline
\end{tabular}

Fuente: elaboración propia, UNED, 2015.

\section{b. Actividad evaluativa}

Se analiza si la cantidad de actividades evaluativas utilizadas influyen en el rendimiento de aprobación, reprobación o deserción de los estudiantes en ambas asignaturas (cuadro 3).

Rendimiento histórico de las asignaturas Globalización y Ambiente y del El Ser Humano en su

Entorno de los años 2007-2015.

Verónica Bonilla-Villalobos

DOI: http://dx.doi.org/10.22458/caes.v8i2.1923

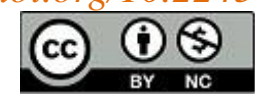


Cuadro 3: Cantidad de instrumentos por cuatrimestres que se utiliza por asignatura

\begin{tabular}{|c|c|c|c|c|c|c|}
\hline \multicolumn{7}{|c|}{ Actividad evaluativa de las asignaturas } \\
\hline Año & \multicolumn{3}{|c|}{ Globalización y Ambiente } & \multicolumn{3}{|c|}{ El Ser Humano en su Entorno } \\
\hline & $\mathrm{I}$ & II & III & 1 & II & \\
\hline 007 & - & - & - & - & & $\begin{array}{l}\text { I y II Ord. } \\
\text { Un Proyecto de }\end{array}$ \\
\hline 2008 & & & & I Proyec & & \\
\hline & - & - & - & $\begin{array}{c}\text { de } \\
\text { investigación } \\
\text { I y II Tarea }\end{array}$ & - & $\begin{array}{l}\text { I, II, III y IV } \\
\text { Tareas }\end{array}$ \\
\hline 2009 & - & - & - & $\begin{array}{l}\text { I y || Ord. } \\
\text { | y || Tarea }\end{array}$ & - & $\begin{array}{l}\text { I y || Ord. } \\
\text { | y || Tarea }\end{array}$ \\
\hline 2010 & - & - & - & - & $\begin{array}{l}\text { I y || Ord. } \\
\text { | y || Tarea }\end{array}$ & \\
\hline 2011 & - & - & - & - & $\begin{array}{l}\text { | y || Ord. } \\
\text { | y || Tarea }\end{array}$ & $\begin{array}{l}\text { I y || Ord. } \\
\text { | y || Tarea }\end{array}$ \\
\hline 2012 & - & - & $\begin{array}{l}\text { I y II Ord. } \\
\text { I Proyecto de } \\
\text { investigación }\end{array}$ & - & $\begin{array}{l}\text { | y || Ord. } \\
\text { | y || Tarea }\end{array}$ & $\begin{array}{l}\text { I y }|| \mathrm{O} \\
\text { | y }|| \mathrm{Ta}\end{array}$ \\
\hline 2013 & $\begin{array}{l}\text { I y || Ord. } \\
\text { I Proyecto } \\
\text { de } \\
\text { investigación }\end{array}$ & $\begin{array}{c}\text { I y II Ord. } \\
\text { I Proyecto } \\
\text { de } \\
\text { investigación }\end{array}$ & & $\begin{array}{c}\text { I y II Ord. } \\
\text { I y II Tarea } \\
\text { I Proyecto } \\
\text { de } \\
\text { investigación }\end{array}$ & $\begin{array}{c}\text { I y II Ord. } \\
\text { I Proyecto } \\
\text { de } \\
\text { investigación }\end{array}$ & $\begin{array}{l}\text { I y || Ord. } \\
\text { I Proyecto de } \\
\text { investigación }\end{array}$ \\
\hline 201 & $\begin{array}{c}\text { I y II Ord. } \\
\text { I Proyecto } \\
\text { de } \\
\text { investigación }\end{array}$ & $\begin{array}{l}\text { I y II Ord. } \\
\text { I Proyecto } \\
\text { de } \\
\text { investigación }\end{array}$ & $\begin{array}{c}\text { I y II Ord. } \\
\text { I } \\
\text { Anteproyecto } \\
\text { y } \\
\text { I Proyecto de }\end{array}$ & $\begin{array}{l}\text { I y II Ord. } \\
\text { I Proyecto } \\
\text { de } \\
\text { investigación }\end{array}$ & $\begin{array}{c}\text { I y II Ord. } \\
\text { I Proyecto } \\
\text { de } \\
\text { investigación }\end{array}$ & $\begin{array}{l}\text { I y II Ord. } \\
\text { I Anteproyecto y } \\
\text { I Proyecto de } \\
\text { investigación }\end{array}$ \\
\hline 2015 & $\begin{array}{l}\text { I y || Ord. } \\
\text { I y || Ensayo }\end{array}$ & $\begin{array}{l}\text { I y || Ord. } \\
\text { | y || Ensayo }\end{array}$ & $\begin{array}{l}\text { I y || Ord. } \\
\text { | y || Ensayo }\end{array}$ & $\begin{array}{l}\text { I y || Ord. } \\
\text { I y || Ensayo }\end{array}$ & $\begin{array}{l}\text { I y || Ord. } \\
\text { I y || Ensayo }\end{array}$ & $\begin{array}{l}\text { I y || Ord. } \\
\text { | y || Ensayo }\end{array}$ \\
\hline
\end{tabular}

Fuente: elaboración propia, información obtenida de orientaciones académicas, UNED, 2015.

Rendimiento histórico de las asignaturas Globalización y Ambiente y del El Ser Humano en su

Entorno de los años 2007-2015.

Verónica Bonilla-Villalobos

DOI: http://dx.doi.org/10.22458/caes.v8i2.1923

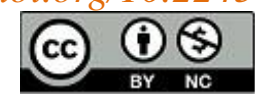


Se determinó que la cantidad de actividad evaluativa de los aprendizajes por cuatrimestre es de un aproximado de tres a cuatro instrumentos aplicados; por ejemplo, en Globalización y Ambiente en un periodo de cinco (5) cuatrimestres se utilizaron tres (3) actividades evaluativas y solamente en un cuatrimestre se usaron cuatro instrumentos. En El Ser Humano en su Entorno en seis (6) cuatrimestres se utilizaron tres (3) instrumentos y en los otros nueve (9) se aplicaron cuatro (4) instrumentos, solamente en uno se utilizaron cinco (5).

\section{Rendimiento de los estudiantes por asignatura}

El Ser Humano en su Entorno: En primer lugar, se realizó una revisión de la matrícula y posteriormente del rendimiento de los estudiantes desde el 2007 al 2015, según categorías (cuadro 5).

Cuadro 5. Cantidad de estudiantes según categorías 2017-2015

\begin{tabular}{c|c|c|c|c}
\hline Año & Cant. Est. & Aprobados & Reprobada & Otros $^{*}$ \\
\hline 2007 & 32 & 27 & 3 & 2 \\
2008 & 114 & 90 & 9 & 15 \\
2009 & 121 & 95 & 13 & 13 \\
2010 & 326 & 241 & 63 & 22 \\
2011 & 1980 & 1213 & 517 & 250 \\
2012 & 2200 & 1415 & 538 & 247 \\
2013 & 3090 & 1904 & 913 & 273 \\
2014 & 3015 & 1947 & 725 & 343 \\
2015 & 3039 & 1898 & 879 & 262 \\
\hline
\end{tabular}

Fuente: elaboración propia, datos compilados del CIEI, UNED, 2015.

\section{Rendimiento histórico de las asignaturas Globalización y Ambiente y del El Ser Humano en su \\ Entorno de los años 2007-2015. \\ Verónica Bonilla-Villalobos \\ DOI: http://dx.doi.org/10.22458/caes.v8i2.1923}

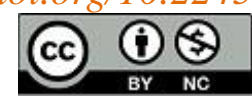


Los resultados muestran un incremento en la cantidad de estudiantes matriculados durante los años 2007-2015, lo anterior debido a la periodicidad de la oferta, los primero años de su oferta se evidencia un porcentaje de aprobación entre 74 y $84 \%$ (figura 3).

Figura 3: Cantidad de estudiantes por año, aprobados, reprobados y otros

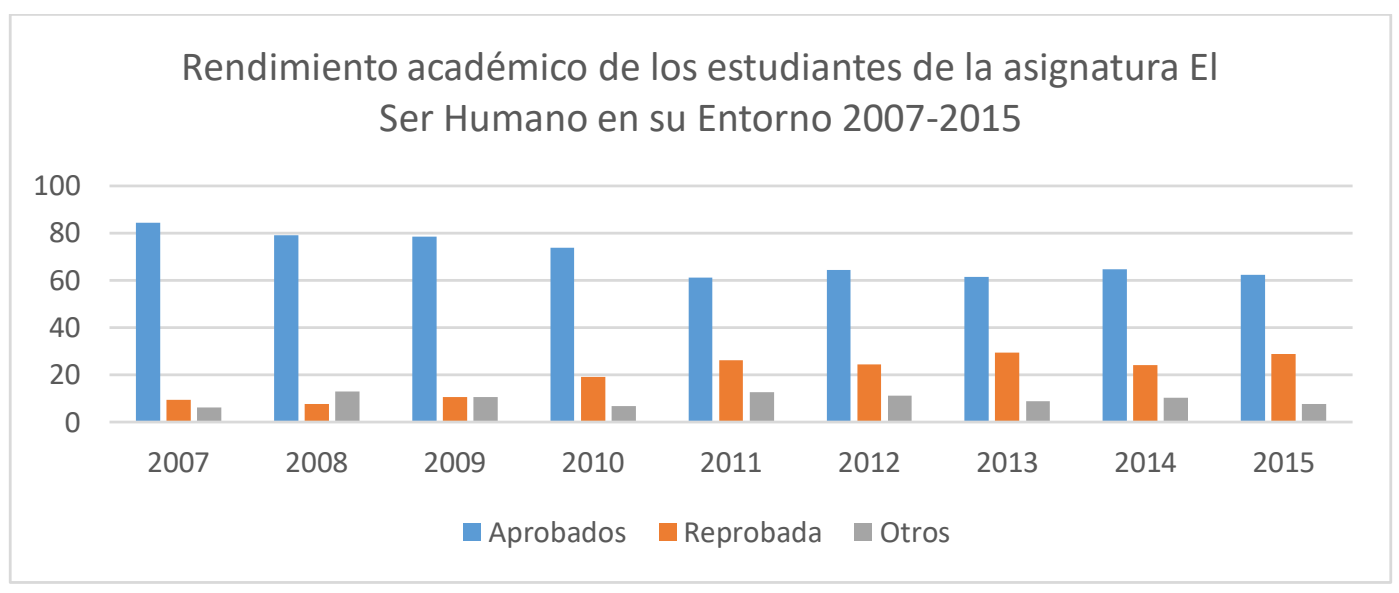

Fuente: elaboración propia, datos compilados del CIEI, UNED, 2015.

Es importante destacar que esta asignatura se ofertó dos veces al año el 2008- 2009 y 2011 2012; en cuanto al retiro injustificado y justificado (serie denominada otros); presentan porcentajes similares $13 \%$ y $11 \%$ los más altos.

En la figura 4, se puede observar que para el periodo del 2007 al $201564 \%$ de los estudiantes aprobaron la asignatura, mientras $26 \%$ la reprobó.

\footnotetext{
Rendimiento histórico de las asignaturas Globalización y Ambiente y del El Ser Humano en su

Entorno de los años 2007-2015.

Verónica Bonilla-Villalobos

DOI: http://dx.doi.org/10.22458/caes.v8i2.1923
}

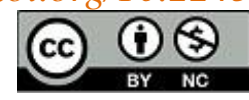

Artículo protegido por licencia Creative Commons 
Figura 4. Cantidad de estudiantes según categoría durante los años 2007-2015

Promedios de aprobación de la asignatura El Ser

Humano en su Entorno 2007-2015

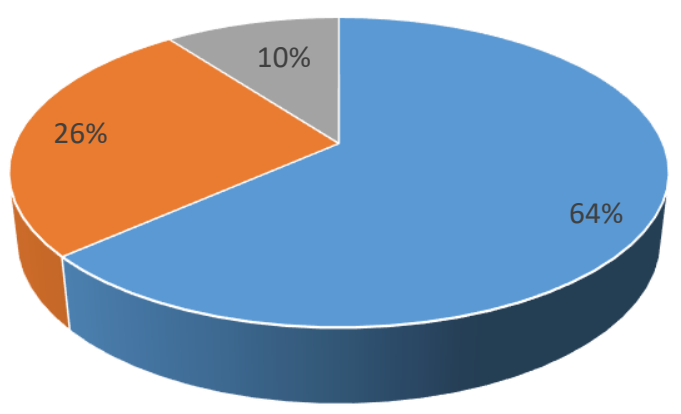

Fuente: elaboración propia, datos compilados del CIEI, UNED, 2015.

En cuanto al promedio de aprobación se encontró que del 2011 al 2015 el promedio oscila entre 62\% y 65\%; mostrando una gran diferencia entre el 2007 al 2010, donde se destaca el 2007 como el año de mayor aprobación (84\%).

En cuanto al promedio de reprobados se identificó que en el 2013 es el más alto con 30\%, seguido del 2015 con 29\%; 2011 con 26\%; 2012 y 2014 con 24\%. Se determinó no vinculante la cantidad de instrumentos de evaluación de los aprendizaje, para los años anteriormente mencionados se utilizan entre tres y cuatro instrumentos; sin embargo, la misma cantidad de actividades se trabajan en otros años y el porcentaje de reprobados difiere.

Rendimiento histórico de las asignaturas Globalización y Ambiente y del El Ser Humano en su

Entorno de los años 2007-2015.

Verónica Bonilla-Villalobos

DOI: http://dx.doi.org/10.22458/caes.v8i2.1923

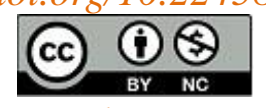

Artículo protegido por licencia Creative Commons 
Globalización y Ambiente: Se identificó un aumento progresivo de la matrícula desde el 2012 al 2015; para el primer año se oferta un cuatrimestre, para el segundo año dos; no obstante, a partir del año 2014 se oferta tres veces (cuadro 4).

Cuadro 4. Cantidad de estudiantes según categorías en los años 2012-2015

\begin{tabular}{c|c|c|c|c}
\hline Año & Cant. Est. & Aprobados & Reprobada & Otros* $^{*}$ \\
\hline 2012 & 407 & 288 & 82 & 37 \\
2013 & 1586 & 1135 & 285 & 166 \\
2014 & 2512 & 1630 & 615 & 267 \\
2015 & 2544 & 1572 & 780 & 192 \\
\hline \multicolumn{2}{l}{ Nota: Otros ${ }^{*}$ (Retiros justificados/ injustificados/reconocidas/ en curso) } \\
\hline
\end{tabular}

Fuente: elaboración propia, datos compilados CIEI, 2015.

Los resultados evidencian que en los años 2012 y 2013 hay un porcentaje de aprobación de $72 \%$, mientras 2014 y 2015 presentan una disminución y un promedio de aprobación de 65\%; sin embargo, es importante indicar que desde el 2015 se disminuyeron los retiros injustificados (figura 5).

Rendimiento histórico de las asignaturas Globalización y Ambiente y del El Ser Humano en su

Entorno de los años 2007-2015.

Verónica Bonilla-Villalobos

DOI: http://dx.doi.org/10.22458/caes.v8i2.1923

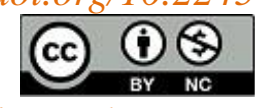


Figura 5. Cantidad de estudiantes por año, aprobados y reprobados 2012-2015

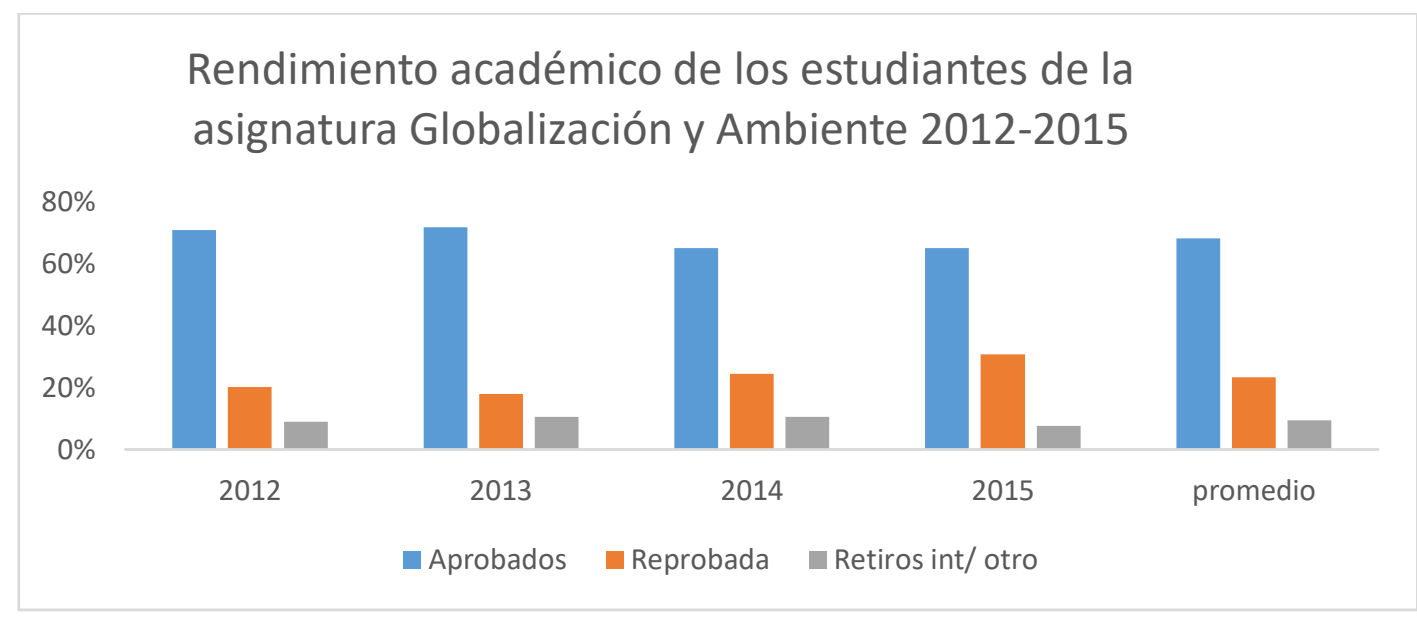

Fuente: Elaboración propia a partir de los datos Cátedra Sociedad y Ambiente, 2015.

En el 2014 y 2015 se refleja un aumento del porcentaje de estudiantes reprobados 24 y $31 \%$, respectivamente.

En la figura 6 se muestra un promedio de $68 \%$ de aprobación y un 23\% de reprobados en la asignatura Globalización y Ambiente durante el periodo 2012 al 2015.

Rendimiento histórico de las asignaturas Globalización y Ambiente y del El Ser Humano en su

Entorno de los años 2007-2015.

Verónica Bonilla-Villalobos

DOI: http://dx.doi.org/10.22458/caes.v8i2.1923

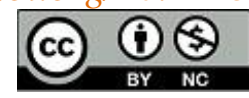

Artículo protegido por licencia Creative Commons 
Figura 6. Rendimiento de los estudiantes en la asignatura Globalización y Ambiente 20122015.

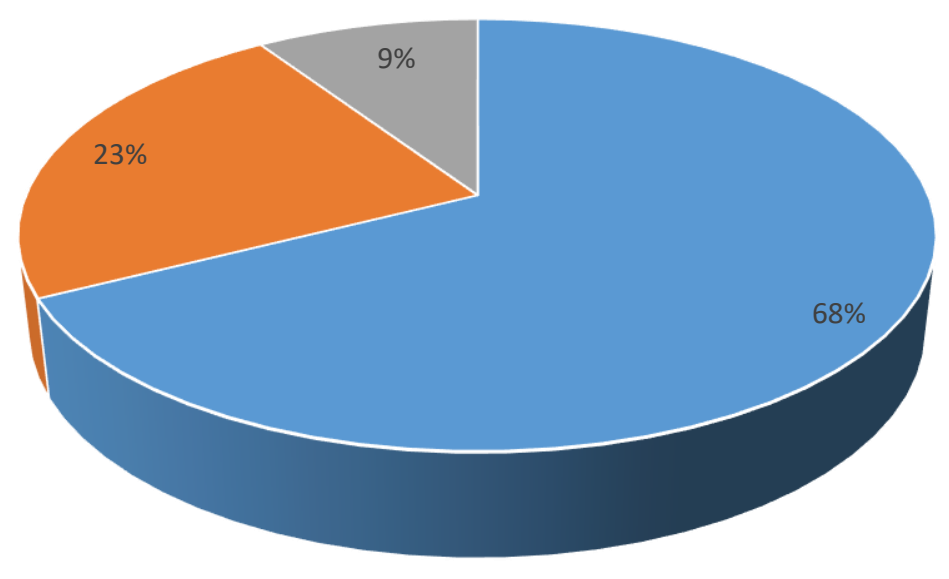

- Aprobados

- Reprobada

- Retiros int/ otro

Fuente: Elaboración propia a partir de los datos Cátedra Sociedad y Ambiente, 2015.

\section{Conclusiones}

En relación con la asignatura 073, se muestra una marcada diferencia entre los años 2007 al 2010 y del 2011 al 2015 en cuanto a porcentajes de aprobación; sin embargo, se identifica que variación del promedio no está relacionada directamente con la cantidad de actividades que el estudiante realizó.

Durante el 2008, 2009, 2011 y 2012 la asignatura se ofertó dos veces al año, en los dos primeros el porcentaje de aprobación fue de 79\%, mientras que para el 2011 bajo a $61 \%$ y

\footnotetext{
Rendimiento histórico de las asignaturas Globalización y Ambiente y del EI Ser Humano en su

Entorno de los años 2007-2015.

Verónica Bonilla-Villalobos

DOI: http://dx.doi.org/10.22458/caes.v8i2.1923
}

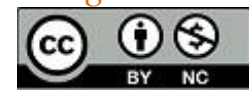


para el 2012 se presentó un aumento de matrícula de 220 estudiantes de más, lo que generó $64 \%$ de aprobación.

Se encontró que en el año 2008 la asignatura 073 no presenta pruebas escritas, no obstante, en el 2009 se incorporan dos pruebas escritas, ante estas dos variables el promedio de aprobación es el mismo $79 \%$.

En síntesis, tanto la asignatura El Ser Humano en su Entorno como Globalización y Ambiente, muestran una constante en el promedio de estudiantes aprobado los primeros años, pero disminuyó posteriormente sin mostrar relación con la cantidad de instrumentos de evaluación.

El promedio de aprobación general durante el periodo de la investigación para El Ser Humano en su Entorno es de 70\% y para Globalización de 68\%; y en cuanto a porcentajes de reprobados es de $20 \%$ y $23 \%$ respectivamente, el restante porcentaje corresponde a otros rubros. Al ser estas asignaturas de estudiantes de primer ingreso en la institución es un porcentaje aceptable, tomando en cuenta que es el primer acercamiento con el sistema de estudios a distancia.

Por otro lado, el cambio de la modalidad de evaluación que se realizó para el III cuatrimestre del 2014, donde se incluía un anteproyecto como retroalimentación para los estudiantes, lo cual no fue del todo satisfactorio ya que algunos no presentaban el anteproyecto y tampoco lo adjuntaban al proyecto final para ver los cambios, por tanto, no se cumplió el objetivo de retroalimentación.

El modelo se ajusta nuevamente para el 2015 , se nota un cambio muy poco significativo en cuanto a rendimiento general de estudiantes aprobados; decayó en el 2015 disminuye el

Rendimiento histórico de las asignaturas Globalización y Ambiente y del El Ser Humano en su

Entorno de los años 2007-2015.

Verónica Bonilla-Villalobos

DOI: http://dx.doi.org/10.22458/caes.v8i2.1923

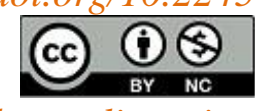

Artículo protegido por licencia Creative Commons 
porcentaje de retiros. Se reevalúan los actividades evaluativas, tomando en cuenta las observaciones de los profesores tutores, sin embargo, al realizar los trabajos de manera individual la carga de trabajo de los tutores es más alta y los tiempos de revisión son los mismos (se puede crear subjetividad por el grado de cansancio a la hora de la revisión).

La Cátedra con esta investigación contribuye con una revisión de los hallazgos del comportamiento de los estudiantes en un periodo de tiempo, lo anterior con el fin de ir en búsqueda de la calidad y de un buen rendimiento académico tal y como lo demuestra Garbanzo (2007) quien indica la necesidad de una revisión integral sobre el rendimiento académico del alumnado; por tanto los resultados son un insumo importante, pues permiten conocer elementos obstaculizadores y facilitadores del desempeño estudiantil, en consecuencia, permiten favorecer el control de los recursos estatales y la mediación del impacto social.

La investigación también identificó en cuáles centros universitarios el rendimiento académico tiende a bajar, no obstante, para conocer los aspectos que inciden en esto, se deberá aplicar otros tipos de herramientas en futuras investigaciones.

\section{Recomendaciones}

La planificación y el diseño de estas asignaturas deben:

- Contemplar las características y necesidades de los contextos socioculturales, económicos y ambientales.

- Implementar una medición pedagógica asertiva en los procesos de comunicación presentes entre el docente y el estudiantado.

- Identificar cuáles son las herramientas más oportunas para lograr con éxito la mediación, contemplando la cantidad de estudiantes.

Rendimiento histórico de las asignaturas Globalización y Ambiente y del El Ser Humano en su

Entorno de los años 2007-2015.

Verónica Bonilla-Villalobos

DOI: http://dx.doi.org/10.22458/caes.v8i2.1923

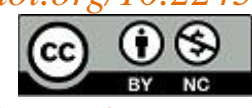

Artículo protegido por licencia Creative Commons 
- Tomar en cuenta la accesibilidad al internet en todas las áreas del país y en los centros universitarios y buscar alternativas de acceso.

- Capacitar a los tutores en el diseño y la participación en las plataformas virtuales, tomando en cuenta que la mayoría son tutores regionales (fuera del área metropolitana) y la UNED representa su segundo trabajo.

- Promover una mayor participación de los tutores en actividades pedagógicas y de estrategias de aprendizaje con el fin de que sean propositivos e innovadores en el uso de las tecnologías de la información y comunicación.

- Crear proyectos pilotos en los cuales se establezca la oferta bimodal; es decir, que se ofrezca en dos modalidades regular e híbridas en los centros universitarios específicos (es importante tomar en cuenta los recursos tecnológicos de cada uno), con el fin de medir la participación e identificar los porcentajes de aprobación de los estudiantes.

Las experiencias que posee la UNED con la utilización de las TIC son excelente con la facilidad de que la cantidad de estudiantes es pequeña y se logra una comunicación tanto sincrónica como asincrónica. Por último, se recomienda promover un análisis por centros universitarios de la UNED para conocer cuáles son los motivos de la baja deserción y reprobación de estas asignaturas.

\section{Referencias}

Castaño, E., Gallón, S., Gómez, K., \& Vásquez, J. (2008). Análisis de los factores asociados a la deserción estudiantil en la Educación Superior: un estudio de caso Analysis of the Factors Associated with the Drop-out Rate of Students in Higher Education: a Case Study. Revista de Educación,345, 255-280.

Rendimiento histórico de las asignaturas Globalización y Ambiente y del El Ser Humano en su

Entorno de los años 2007-2015.

Verónica Bonilla-Villalobos

DOI: http://dx.doi.org/10.22458/caes.v8i2.1923

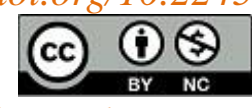

Artículo protegido por licencia Creative Commons 
Colmenares, M. \& Delgado, F. (2008). Aproximación teórica al estado de la relación entre rendimiento académico y motivación de logro en educación superior. Revista de Ciencias Sociales, 4(3), $604-613$

Driscoll, M. (2005). Psychology of Learning for instructions (3era ed.). Needham Heights, MA: Allyn and Bacon.

Fallas, I. y Trejos, I. (2013). Educación en la Sociedad de la información y el conocimiento, Cap. 2. Tecnología educativa, paradigma y cambio, EUNED, UNED, Costa Rica.

Nolla Cao, N. (1998). Modelo de evaluación de un plan de estudios para las especialidades médicas y estomatológicas. Educación Médica Superior, 12(2), 62-72.

Universidad Estatal a Distancia (UNED).(2014). Escuela de Ciencias Sociales y Humanidades, ECSH 2014, Programa de Estudios Generales, San José, Costa Rica.

Vargas, G. (2007). Factores asociados al rendimiento académico en estudiantes universitarios, una reflexión desde la calidad de la educación superior pública. Revista educación, 31(1), 43-63.

Rodríguez, A., \& Vindas, M. (2005). La deserción estudiantil en la educación superior: el caso de la Universidad de Costa Rica. Actualidades investigativas en educación,5(4).1-22

Rendimiento histórico de las asignaturas Globalización y Ambiente y del El Ser Humano en su

Entorno de los años 2007-2015.

Verónica Bonilla-Villalobos

DOI: http://dx.doi.org/10.22458/caes.v8i2.1923

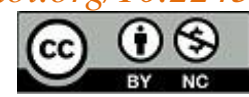

